\title{
Cryptographic Protocols for Enforcing Relationship-based Access Control Policies
}

\author{
Jun Pang* ${ }^{* \dagger}$, Yang Zhang ${ }^{\dagger}$ \\ *Interdisciplinary Centre for Security, Reliability and Trust, University of Luxembourg \\ ${ }^{\dagger}$ Faculty of Science, Technology and Communication, University of Luxembourg
}

\begin{abstract}
Relationship-based access control schemes have been studied to protect users' privacy in online social networks. In this paper, we propose cryptographic protocols for decentralized social networks to enforce relationship-based access control polices, i.e., k-common friends and k-depth. Our protocols are mainly built on pairing-based cryptosystems. We prove their security under the honest but curious adversary model, and we analyze their computation and communication complexities. Furthermore, we evaluate their efficiency through simulations on a real social network dataset.
\end{abstract}

\section{INTRODUCTION}

Online social networks (OSNs) have gained a huge success during the past decade. Nowadays, using OSNs service almost becomes an indispensable part in people's daily lives. A user in OSNs can specify his profile, articulate his social relationships, share his life moments, etc. With more and more personal information appearing online, users' privacy has become an essential problem since, as in most of the times users don't want to share their lives with everybody. Access control is one of the most straightforward but useful ways to address this challenge. In recent years, industries have adopted relationship-based access control schemes for OSNs, for example, Facebook and LinkedIn. In such schemes, whether a user can view another user's information depends on the relationship between these two users. For example, a user in Facebook can decide which users can view his photos, based on their social depth to himself - being his friends, friends of friends, etc. However, social relationships as well as interactions among people are very complicated, they cannot be fully represented by "friends" and "friends of friends". For example, if two strangers share a lot of common friends, then the chance that they trust each other is high [1]. Thus, it is more probable that they want to interact with each other. On the other hand, if these two users only have one friend in common, even though their relationship is "friend of friend", the chance for them to communicate is much lower than the former case. Situations like this happen often but the current access control schemes in OSNs do not support them. Therefore, it is necessary to have more fine-grained access control schemes.

Several fine-grained relationship-based access control polices have been proposed by research communities [2], [3], [4]. These policies are necessary as they enable users to have more precise and strict control on who can access their personal information or resources. On the other hand, such policies are quite flexible and give users possibilities to regulate access control based on relationships and the topology of the underlying social graph. For instance, a user can define a policy allowing the users who have at least a number of common friend with him to review his photos. The user can also specify a policy that only his friends and friends of his family members can view his photos. Despite of their expressiveness and flexibility, implementing these policies normally requires many computing resources which are usually the bottleneck even for big companies, like Facebook and Google. Decentralized social networks (e.g., [5], [6]) have been proposed in the literature as an ideal solution to address the problem. In decentralized social networks, users can control their own data and enforce access control polices with their personal devices instead of putting the burden on the central operators' shoulder [7]. In this way, users can get rid of the central operators and implementing fine-grained relationship-based access control polices will then only involve social network users. In recent years, developing cryptographic protocols for enforcing fine-grained access control polices in decentralized social networks has been an active research area (see more discussions in Section II).

Contributions. In this paper, we propose cryptographic protocols to implement two fine-grained access control polices, i.e., ' $k$-common friends' and ' $k$-depth' as proposed in [4], for decentralized social networks. Our main contributions in this paper are summarized as follows.

- We propose the first protocol to enforce $k$-common friends policies. Our construction is based on paring-based cryptosystems (PBC) and private set intersection protocols. Security analysis shows that our protocol is secure under the honest but curious adversary model.

- Our protocol for $k$-depth policies is based on PBC as well. We have proved that our protocol is secure under the honest but curious adversary model. Compared to existing protocols, our protocol achieves a better security level. Our $k$-depth protocol can be extended to support multirelationship social networks.

- For our protocols, we perform a detailed analysis of their efficiency and conduct an empirical evaluation of their performance with a real-life social network dataset. The results show that our protocols are quite practical. 
Organization. After presenting related work in Section II, we introduce some preliminaries in Section III. The OSN model as well as access control polices are presented in Section IV. Our protocols for enforcing two relationship-based access control policies are described in Section $\mathrm{V}$ and Section VI together with corresponding security proofs, respectively. The theoretical as well as empirical evaluation of our protocols are presented in Section VII. Section VIII concludes the paper with some future work.

\section{RELATED WORK}

Access control models. Carminati et al. [8] proposed the first relationship-based access control model where polices are based on three regulations including relationship type, depth and trust value. In [3], a fine-grained model based on semantic web tools is introduced, where they proposed 'admin' and 'filtering' polices. Fong et al. introduced an access control scheme which supports Facebook-style social networks [4]. Specifically, they proposed a few fine-grained access control polices including the topology-based ones that we will focus on in this paper. In [9], [10], [11], modal (hybrid) logics are exploited to express access control polices. Recently, Pang and Zhang [12] extended their work by defining a new OSN model containing users and their relationships as well as public information. Based on this new model, they introduced a new hybrid logic for formulating access control policies. Cheng et al. [13] presented a social network model where resources are also treated as nodes. With this model, access control polices, related to not only user-to-user but also user-to-resource and resource-to-resource relationships, are supported.

Security protocols. Besides access control models, security protocols for enforcing access control polices in OSNs have also been studied. Carminati et al. introduced several solutions [14], [15], [16]. For instance, in [16], a homomorphic encryption scheme is used to compute aggregated information of the path (trust level and relationship type), which minimizes the loss of sensitive information. In [17], Mezzour et al. proposed an interesting solution for path discovery where the protocol contains two stages - in the first stage, each user floods tokens in the social network, while in the second stage a private set intersection protocol is executed for finding paths. Backes et al. [18] defined a security API for distributed social networks, where cryptographic techniques such as pseudonyms, digital signatures and zero-knowledge proofs are exploited to help user to establish and prove the existence of friendships with others. In [19], a key management scheme was proposed under which only users who are within a certain distance to the owner are able to derive keys to decrypt the encrypted resources. A comparison between our protocols and two existing protocols is presented in Section VII.

\section{PRELIMINARIES}

\section{A. Cryptographic Building Blocks}

Bilinear map. Let $G_{1}=\langle g\rangle$ and $G_{2}$ be two multiplicative groups of the same prime order $p$. An efficient computable map $e: G_{1} \times G_{1} \rightarrow G_{2}$ is a bilinear map if the following properties hold:

$$
\begin{array}{ll}
\text { Bilinearity: } & \forall a, b \in \mathbb{Z}_{p}^{*}, e\left(g^{a}, g^{b}\right)=e(g, g)^{a b} \\
\text { Non-Degeneracy: } & e(g, g) \text { is a generator of } G_{2}
\end{array}
$$

Computational Diffie-Hellman (CDH) problem. This problem states that given $g^{a}, g^{b} \in G_{1}$, find $g^{a b} \in G_{1}$. CDH assumption means that there is no probabilistic polynomial time algorithm to solve $\mathrm{CDH}$ problem in $G_{1}$.

A variant of $\mathrm{CDH}$ problem is called Reversion Computational Diffie-Hellman (RCDH) problem: given $g^{a}, g^{c}$, find $g^{c / a}$. In [20], the authors proved that $\mathrm{RCDH}$ problem is equivalent to $\mathrm{CDH}$ problem.

Due to the existence of bilinear map $e$, Decisional DiffieHellman (DDH) problem, i.e., given $g^{a}, g^{b}, g^{c} \in G_{1}$, decide whether $g^{a b}=g^{c}$ or not, can be efficiently solved in $G_{1}$ while $\mathrm{CDH}$ problem remains hard. $G_{1}$ is also referred as a Gap Diffie-Hellman (GDH) group.

Bilinear Diffie-Hellman (BDH) problem. It can be considered as a $\mathrm{CDH}$ problem in $G_{2}$. It states that, given $g^{a}, g^{b}, g^{c} \in$ $G_{1}$, find $e(g, g)^{a b c} \in G_{2}$. Again, BDH assumption indicates that there is no probabilistic polynomial time algorithm that can solve BDH problem in $G_{2}$.

BLS signature. Boneh et al. [21] proposed a short signature scheme based on GDH groups. An approximately 160-bit BLS signature can achieve a similar security level of a 320bit DSA signature. The BLS signature scheme contains three algorithms, i.e., KeyGen, Sign and Verify, and hash function $H:\{0,1\}^{*} \rightarrow G_{1}$ is a random oracle [22].

KeyGen. Each party chooses a random value $x$ from $\mathbb{Z}_{p}^{*}$ (denoted by $x \stackrel{r}{\leftarrow} \mathbb{Z}_{p}^{*}$ ) as its private key; the corresponding public key is $g^{x} \in G_{1}$.

Sign. To sign a message $m$, the signer hashes $m$ into $G_{1}$, i.e., $H(m)$, and computes $H(m)^{x}$.

Verify. Given $g^{x}, H(m)^{x}$ and $m$, the verifier computes $H(m)$ and checks if $e\left(H(m)^{x}, g\right)=e\left(H(m), g^{x}\right)$ holds.

Private set intersection. A private set intersection (PSI) protocol allows two parties to find the intersection of their input sets without leaking extra information (e.g., see [23], [24], [25]). A cardinality PSI protocol only allows two parties to learn the size of the intersection of their sets. In our work, we exploit a cardinality PSI protocol which is secure against the honest but curious adversary (which we will introduce later) and treat it as a black box.

\section{B. Adversary Model}

In this paper, we focus on the honest but curious adversary model and its detailed formal definitions can be found in [26]. Under this model, all users follow the specified protocol. An adversary tries to get some additional knowledge by inspecting the protocol transcripts that he gets after the protocol execution. To illustrate the security of our proposed protocols under this model, we show that a party cannot get any extra information with the protocol transcripts as well as the outputs. Note that we assume communication channels among parties are authenticated, i.e., impersonating attacks are not possible. 


\section{OnLINE Social Networks}

\section{A. Social Network Model}

We model a social network as a social graph $\mathcal{G}=(\mathcal{U}, \mathcal{E})$ where each user $u_{i} \in \mathcal{U}$ is represented as a single node and social relationships among them are represented in the form of edges $(\mathcal{E})$. Without ambiguity, we directly use $u_{i}$ to represent $u_{i}$ 's identity. We first only consider friend relationships, i.e., friendships, in our model. It is not difficult to extend the proposed protocols for multi-relationships. $\mathcal{E}$ is defined as a subset of $\mathcal{U} \times \mathcal{U}$, i.e., when two users $u_{i}$ and $u_{j}$ establish a friendship, an edge between them is added into $\mathcal{G}$. The set $u_{i}$.fri contains all $u_{i}$ 's friends. When $u_{i}$ and $u_{j}$ are friends, we have $u_{j} \in u_{i}$.fri and $u_{i} \in u_{j}$.fri. A path from one user to another in $\mathcal{G}$ is represented by a sequence of users on this path, the number of edges on this path is defined as its depth. For example, a path from $u_{i}$ to $u_{j}$ with $u_{\ell}$ as the middle node is denoted by $\left[u_{i}, u_{\ell}, u_{j}\right]$ and it is a 2 -depth path. Here, $u_{i}$ is also referred as the originator of the path. Moreover, two paths are reverse for each other if they have same users but in reverse sequences, e.g., $\left[u_{j}, u_{\ell}, u_{i}\right]$ is a reverse path of $\left[u_{i}, u_{\ell}, u_{j}\right]$.

Besides social information, each user is equipped with some algebraic knowledge. The two previously mentioned groups $G_{1}=\langle g\rangle$ and $G_{2}$ of the same prime order $p$ together with a bilinear map $e: G_{1} \times G_{1} \rightarrow G_{2}$ and a random hash function $H:\{0,1\}^{*} \rightarrow G_{1}$ are publicly known to everyone. A key management authority assigns each user $u_{i}$ a key pair $\left(p k_{i}, s k_{i}\right)$ where the secret key is $s k_{i} \stackrel{r}{\leftarrow} \mathbb{Z}_{p}^{*}$ and its corresponding public key is $p k_{i}=g^{s k_{i}}$. When $u_{i}$ and $u_{j}$ become friends, $u_{i}$ generates a signature $\tau_{j i}=H\left(f r i, u_{j}\right)^{s k_{i}}$ for $u_{j}$ as a friendship certificate. At the same time, $u_{j}$ also issues $u_{i}$ a friendship certificate $\tau_{i j}=H\left(f r i, u_{i}\right)^{s k_{j}}$. The reason to put the identity of the user inside the certificate is to prevent users to transfer their friendship certificates to others. Each user maintains all these certificates as well as the corresponding users' identities who have issued them.

\section{B. Access Control in Social Networks}

In OSNs, a user defines an access control policy to regulate who can view or perform other actions on a certain resource that he has. We refer this user as the owner (represented by $u_{o}$ ) and the user who wants to access the resources as the requester $\left(u_{r}\right)$. We regulate the access control policy on a certain resource is only defined by its owner, i.e., collaborative access control schemes such as [27], [28], [29], [30] are considered out of the scope of our paper. When $u_{r}$ wants to access a certain resource, such as a photo or a status, he first sends $u_{o}$ a request. The owner then extracts the access control policy on that resource and sends it back to $u_{r}$. Next, $u_{o}$ and $u_{r}$ run the protocol related to the policy. In the end, if the result of the protocol shows that $u_{r}$ satisfies the policy's regulation, then $u_{o}$ grants the access of the resource to $u_{r}$. As mentioned in Sect. I, we focus on two access control policies proposed in [4]. We first give their formal definitions as follows.

$k$-common friends. This policy regulates that the qualified requester should have at least $k$ common friends with the owner, formally $\mid u_{o}$. fri $\cap u_{r}$.fri $\mid \geq k$. $k$-depth. This policy specifies that $u_{o}$ is linked with $u_{r}$ through a $k$-depth path.

In the following two sections, we present a protocol for each of the two policies and prove its security under the honest but curious adversary.

\section{V. $k$-COMMON FRIENDS}

\section{A. Protocol Description}

Our solution for $k$-common friends exploits the encoding scheme proposed in [24] and a cardinality PSI protocol.

In the beginning, $u_{r}$ and $u_{o}$ exchange random values with each other, $u_{r}$ sends $R_{r}=g^{r_{r}}$ to $u_{o}$ and $u_{o}$ replies with $R_{o}=g^{r_{o}}$ to $u_{r}$, where $r_{r}, r_{o} \stackrel{r}{\leftarrow} \mathbb{Z}_{p}^{*}$. Next, both parties encode their friendship certificates using Algorithm 1.

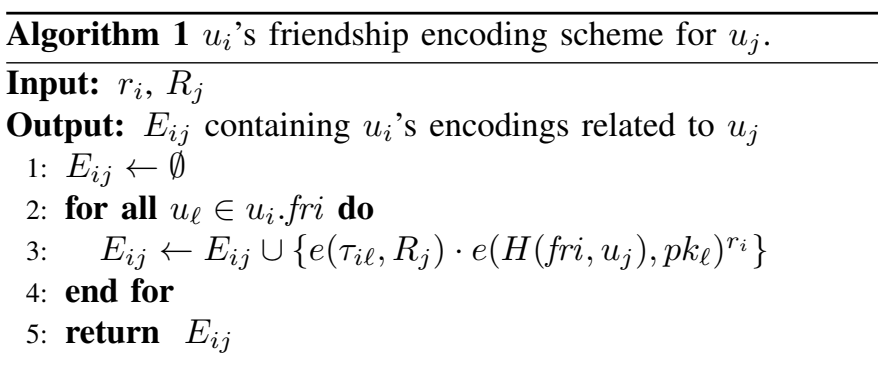

For example, suppose $u_{a} \in u_{o} . f r i \cap u_{r} . f r i, u_{o}$ encodes the certificate $\tau_{o a}=H\left(\text { fri }, u_{o}\right)^{s k_{a}}$ into the following:

$$
e\left(\tau_{o a}, R_{r}\right) \cdot e\left(H\left(f r i, u_{r}\right), p k_{a}\right)^{r_{o}} .
$$

On the other hand, $u_{r}$ encodes $\tau_{r a}=H\left(f r i, u_{r}\right)^{s k_{a}}$ into

$$
e\left(\tau_{r a}, R_{o}\right) \cdot e\left(H\left(f r i, u_{o}\right), p k_{a}\right)^{r_{r}} .
$$

Each encoding contains two components. The first component of (I) is equal to the second component of (II) due to bilinearity of the map $e$, i.e.,

$$
\begin{aligned}
e\left(\tau_{o a}, R_{r}\right) & =e\left(H\left(\text { fri }, u_{o}\right)^{s k_{a}}, g^{r_{r}}\right) \\
& =e\left(H\left(\text { fri }, u_{o}\right), p k_{a}\right)^{r_{r}} .
\end{aligned}
$$

The same with the second component of (I) and the first one of (II). Therefore, encodings (I) and (II) are identical. Actually, the second component of one encoding is an anticipation of the first component of the other one [24]. As long as $u_{o}$ and $u_{r}$ have the proper friendship certificates issued by a common friend, their encodings related to this friend are identical which means they have a common element related to this common friend.

In the end, $u_{o}$ and $u_{r}$ perform a cardinality PSI protocol on these encodings and get the number of their common friends. Note that both $u_{o}$ and $u_{r}$ can choose not to encode their friendships that are considered sensitive for set intersection operations.

All the encodings are based on the friendship certificates which are already part of $u_{o}$ and $u_{r}$ 's knowledge after they establish connections with other users. Therefore, $u_{o}$ and $u_{r}$ 's friends do not need to participate in the process, i.e., the protocol can be executed when they are offline. This is an appealing feature for most situations, as it allows the protocol 


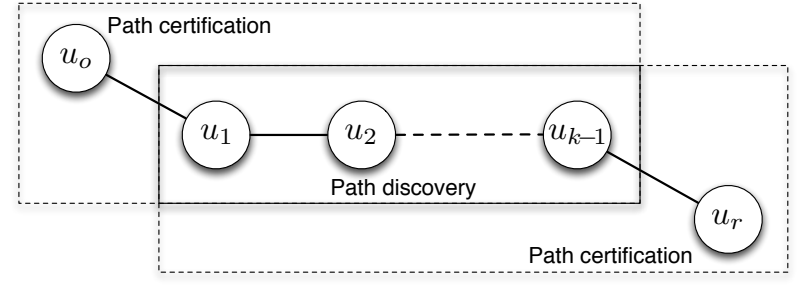

Fig. 1. The two stages of $k$-depth protocol.

exclusively based on $u_{o}$ and $u_{r}$ 's local interaction and without the help of intermediate users.

\section{B. Security Analysis}

The goal of the protocol we want to achieve is that $u_{o}\left(u_{r}\right)$ cannot learn who are friends of $u_{r}\left(u_{o}\right)$. Note that, since $u_{o}$ and $u_{r}$ 's friends don't participate in the protocol, they cannot cause any privacy threat.

In this protocol, except for the PSI operation, $u_{o}$ and $u_{r}$ only perform local computations (with their friendship certificates), i.e., they don't communicate with each other. Therefore, neither of them will get extra information from that stage. As the cardinality PSI protocol we exploit is secure against the honest but curious adversary model, our protocol is secure under this model as well.

\section{VI. $k$-DEPTH}

\section{A. Protocol Description}

The protocol for 2-depth policy can be implemented as a 1-common friend protocol. When the depth is bigger than 2, since neither $u_{o}$ nor $u_{r}$ has information about users beyond their friends, collaboration of intermediate users (neither the owner nor the requester) are needed. In the previous protocol, only $u_{o}$ and $u_{r}$ need to be online, common friends are discovered through friendship certificates that $u_{o}$ and $u_{r}$ have. We apply this idea to obtain our $k$-depth protocol.

Our protocol contains two stages, namely $((k-1)$-depth) path certification and ( $k$-depth) path discovery. As we can see from the two dashed boxes in Figure 1, in the path certification stage, $u_{o}$ and $u_{r}$ ask intermediate users to certify $(k-1)$-depth paths starting from them, respectively. In the path discovery stage, if a $(k-1)$-depth path originated by $u_{o}$ shares a $(k-2)$ depth (reversed) path with a $(k-1)$-depth path originated from $u_{r}$ (see the central solid box in Figure 1), then these two paths can compose a $k$-depth path between $u_{o}$ and $u_{r}$. In our protocol, encodings of these two $(k-1)$-depth paths are identical. After performing a cardinality PSI protocol, a $k$ depth path can be discovered (if such $k$-depth paths exist for $u_{o}$ and $u_{r}$ ).

Besides a key pair, each user $u_{i}$ is also affiliated with a set of depth stamps, i.e., $s_{i}=\left\{s_{i}^{j} \mid s_{i}^{j} \stackrel{r}{\leftarrow} \mathbb{Z}_{p}^{*}\right.$ and $\left.j>0\right\}$, and it is only known to the user himself. Here, $s_{i}^{j}$ is the depth stamp for $u_{i}$ at depth $j$, called $u_{i}$ 's $j$-depth stamp. We regulate that each user's 0 -depth stamp is equal to 1 .
1) Path certification: In this stage, $u_{o}\left(u_{r}\right)$ first invites his friends to join the process by sending them messages. A message $m$ is defined as a tuple $(i, \eta, c n t, d e p)$. Here, the randomly chosen $i$ represents identity of the message; $\eta$ is the path certificate which is equal to $H\left(u_{o}\right)$ for $u_{o}\left(H\left(u_{r}\right)\right.$ for $\left.u_{r}\right)$ at the moment; cnt starting from 1 represents the count value; $d e p=k-1$ is the length of the path. Note that everyone in the path certification stage can choose who to contact next.

Upon receiving a message with $c n t \neq d e p$ from one of his friends, if an intermediate user agrees to join the path certification process, then he follows Algorithm 2.

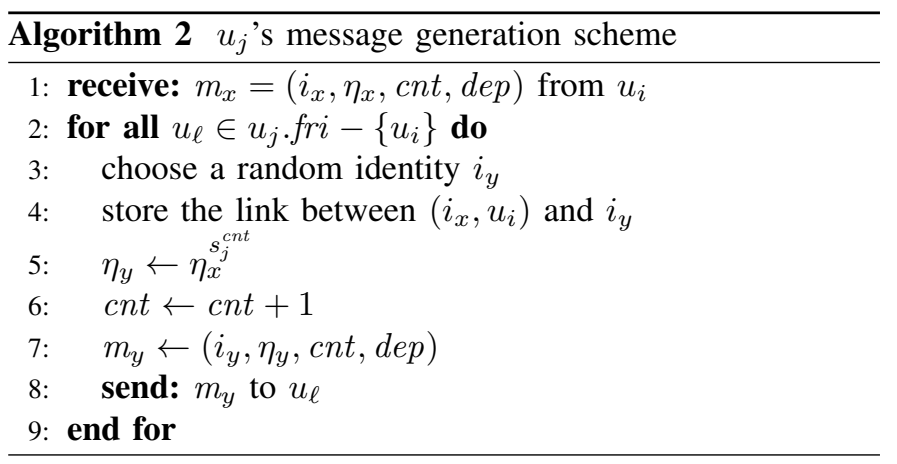

The user first remembers the links between the identity of the received message and identities of new messages that he is going to send out. Next, he generates a new path certificate by raising the old one to the power of his cnt-depth stamp. As the depth stamp is only known to the user, this operation indicates that the user agrees to certify the path. Moreover, by using his $c n t$-depth stamp, the user's position information on the path is directly stored into the certificate. For example, if cnt is equal to 2, then using the user's 2-depth stamp for the new path certificate shows that he is the second one on this path. This is a crucial operation in our protocol. Without it, the result in the path discovery stage may be incorrect, we will show an example later.

If a user receives a message with $c n t=d e p$, then he is aware that he is the last one on the $(k-1)$-depth path. Next, he sends a reverse message back to the friend who sent him the message. The reverse message $\mathrm{rm}$ is also denoted by a tuple $(i, \eta, \sigma, c n t)$ where $i$ is the same as identity of the message he received; $\eta$ is the $(k-1)$-depth path's certificate which will stay the same in the following processes; $\sigma$ represents the path stamp and it is equal to $g^{s_{a}^{1}}$ at the moment if the user is $u_{a}$; cnt is reset to 2 .

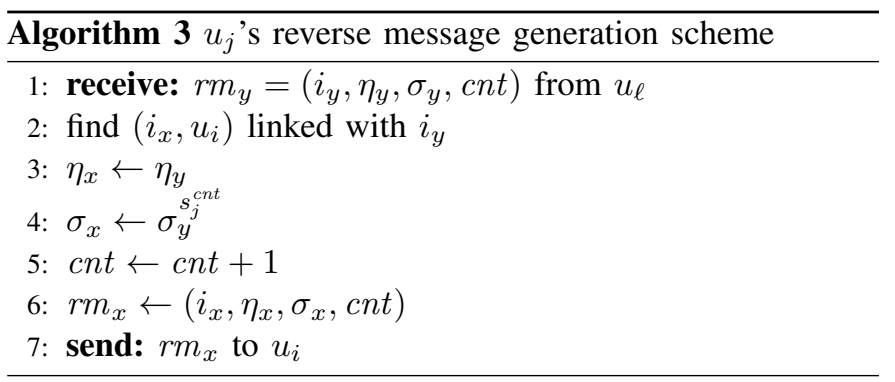

When a user gets a reverse message from his friend, he 
performs the operations as specified in Algorithm 3. Since he has stored the connection between messages' identities, he is able to forward the new reverse message back to the user who sent him the corresponding message previously (step 2 in Algorithm 3). Identity information guarantees that a reverse message's forwarding path is the reverse path of the one on which the corresponding path certificate is established. Each intermediate user also builds the path stamp by raising the old one to the power of the his cnt-depth stamp. Similarly, the sequence of intermediate users are stored into the path stamp. Note that if a user uses his $i$-depth stamp to build a path's certificate, then he computes the path's stamp with his $(k-i)$ depth stamp. Essentially, establishment of a $(k-1)$-depth path's stamp simulates the building process of another $(k-1)$-depth path's certificate where these two paths together compose a $k$-depth path.

In the end, several $(k-1)$-depth path's certificates and stamps are sent back to $u_{o}$ and $u_{r}$.

2) Path discovery: In this stage, $u_{o}$ and $u_{r}$ follow a similar procedure of 1-common friends protocol. First, they exchange two random numbers $R_{o}=g^{r_{o}}$ and $R_{r}=g^{r_{r}}$ while keeping $r_{o}$ and $r_{r}$ secret. Then, as the policy is $k$-depth, they both encode all the $(k-1)$-depth paths' certificates and stamps obtained from the last stage following Algorithm 4.

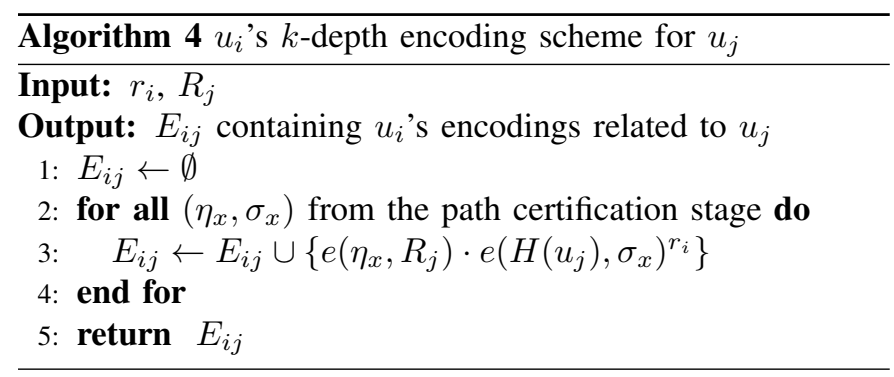

For two $(k-1)$-depth paths starting from $u_{o}$ and $u_{r}$, respectively, if they can compose a $k$-depth path, then exponent of one's stamp is equal to the other one's certificate. Therefore, encodings on certificates and stamps of these two paths will be identical. In the end, a cardinality PSI protocol is performed on these encodings to find out the $k$-depth path.

3) An example: We present an example to show how our $k$-depth protocol works: the network topology is depicted in Figure 2 and the policy is 3-depth.

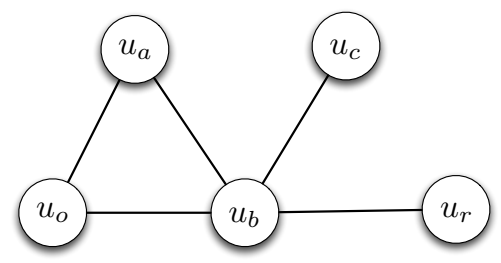

Fig. 2. An example of social network topology.

Path certification stage. In the beginning, $u_{o}$ sends $m_{1}=$ $\left(i_{1}, H\left(u_{o}\right), 1,2\right)$ to $u_{a}$ and $m_{2}=\left(i_{2}, H\left(u_{o}\right), 1,2\right)$ to $u_{b}$. Upon receiving $m_{1}, u_{a}$ chooses a random message identity $i_{3}$ and remembers the link between $\left(i_{1}, u_{o}\right)$ and $i_{3}$. Since $c n t=1$ in $m_{1}$, he computes a new path certificate as $H\left(u_{o}\right)^{s_{a}^{1}}$. Then, $u_{a}$ sends $m_{3}=\left(i_{3}, H\left(u_{o}\right)^{s_{a}^{1}}, 2,2\right)$ to $u_{b}$, i.e., his only friend except $u_{o}$. Meanwhile, $u_{b}$ performs similar operations and sends $m_{4}=\left(i_{4}, H\left(u_{o}\right)^{s_{b}^{1}}, 2,2\right)$ to $u_{a}, m_{5}=\left(i_{5}, H\left(u_{o}\right)^{s_{b}^{1}}, 2,2\right)$ to $u_{c}$ and $m_{6}=\left(i_{6}, H\left(u_{o}\right)^{s_{b}^{1}}, 2,2\right)$ to $u_{r}$.

When $u_{b}$ receives $m_{3}$ and finds out $c n t=d e p$, he knows that he is the last one on the 2-depth path. Since cnt is equal to 2 , he computes $H\left(u_{o}\right)^{1} s_{b}^{2}$ and sends a reverse message $r m_{3}=\left(i_{3}, H\left(u_{o}\right)^{s_{a}^{1} s_{b}^{2}}, g^{s_{b}^{1}}, 2\right)$ back to $u_{a}$. Note that $i_{3}$ in $r m_{3}$ is identical to the identity of $m_{3}$. Similarly, $u_{b}$ gets $r m_{4}=\left(i_{4}, H\left(u_{o}\right)^{s_{b}^{1} s_{a}^{2}}, g^{s_{a}^{1}}, 2\right), r m_{5}=\left(i_{5}, H\left(u_{o}\right)^{s_{b}^{1} s_{c}^{2}}, g^{s_{c}^{1}}, 2\right)$ and $r m_{6}=\left(i_{6}, H\left(u_{o}\right)^{s_{b}^{1} s_{r}^{2}}, g^{s_{r}^{1}}, 2\right)$ from $u_{a}, u_{c}$ and $u_{r}$.

Next, when $u_{a}$ receives $r m_{3}$, he finds out that the identity of the message linked with $i_{3}$ is $\left(i_{1}, u_{o}\right)$. Since cnt is equal to $2, u_{a}$ computes $g^{s_{b}^{1}} s_{a}^{2}$ as a new path stamp and sends $\left(i_{1}, H\left(u_{o}\right)^{s_{a}^{1} s_{b}^{2}}, g^{s_{b}^{1}} s_{a}^{2}, 3\right)$ to $u_{o}$. On the other hand, $u_{b}$ sends $u_{o}$ three reverse messages that have the same identity but with different contents $-\left(i_{2}, H\left(u_{o}\right)^{s_{b}^{1} s_{a}^{2}}, g^{s_{a}^{1} s_{b}^{2}}, 3\right)$, $\left(i_{2}, H\left(u_{o}\right)^{s_{b}^{1} s_{c}^{2}}, g^{s_{c}^{1} s_{b}^{2}}, 3\right)$ and $\left(i_{2}, H\left(u_{o}\right)^{s_{b}^{1} s_{r}^{2}}, g^{s_{r}^{1} s_{b}^{2}}, 3\right)$ to $u_{o}$.

In the end, $u_{o}$ gets four pairs of path certificate and stamp related to four different 2-depth paths starting from him, i.e.,

$$
\begin{aligned}
& \left(H\left(u_{o}\right)^{s_{a}^{1} s_{b}^{2}}, g^{s_{b}^{1} s_{a}^{2}}\right) \text { for path }\left[u_{o}, u_{a}, u_{b}\right] \text {, } \\
& \left(H\left(u_{o}\right)^{s_{b}^{1} s_{a}^{2}}, g^{s_{a}^{1} s_{b}^{2}}\right) \text { for path }\left[u_{o}, u_{b}, u_{a}\right] \text {, } \\
& \left(H\left(u_{o}\right)^{s_{b}^{1} s_{c}^{2}}, g^{s_{c}^{1} s_{b}^{2}}\right) \text { for path }\left[u_{o}, u_{b}, u_{c}\right] \text {, } \\
& \left(H\left(u_{o}\right)^{s_{b}^{1} s_{r}^{2}}, g^{s_{r}^{1} s_{b}^{2}}\right) \text { for path }\left[u_{o}, u_{b}, u_{r}\right] \text {. }
\end{aligned}
$$

On the other direction, $u_{r}$ gets

$$
\begin{aligned}
& \left(H\left(u_{r}\right)^{s_{b}^{1} s_{a}^{2}}, g^{s_{a}^{1} s_{b}^{2}}\right) \text { for path }\left[u_{r}, u_{b}, u_{a}\right] \text {, } \\
& \left(H\left(u_{r}\right)^{s_{b}^{1} s_{c}^{2}}, g^{s_{c}^{1} s_{b}^{2}}\right) \text { for path }\left[u_{r}, u_{b}, u_{c}\right] \text {, } \\
& \left(H\left(u_{r}\right)^{s_{b}^{1} s_{o}^{2}}, g^{s_{o}^{1} s_{b}^{2}}\right) \text { for path }\left[u_{r}, u_{b}, u_{o}\right] \text {. }
\end{aligned}
$$

Path discovery stage. In this stage, $u_{o}$ encodes all 2-depth paths' certificate and stamp into

$$
\begin{aligned}
& e\left(H\left(u_{o}\right)^{s_{a}^{1} s_{b}^{2}}, R_{r}\right) \cdot e\left(H\left(u_{r}\right), g^{s_{b}^{1} s_{a}^{2}}\right)^{r_{o}} ; \\
& e\left(H\left(u_{o}\right)^{s_{b}^{1} s_{a}^{2}}, R_{r}\right) \cdot e\left(H\left(u_{r}\right), g^{s_{a}^{1} s_{b}^{2}}\right)^{r_{o}} ; \\
& e\left(H\left(u_{o}\right)^{s_{b}^{1} s_{c}^{2}}, R_{r}\right) \cdot e\left(H\left(u_{r}\right), g^{s_{c}^{1} s_{b}^{2}}\right)^{r_{o}} \text {; } \\
& e\left(H\left(u_{o}\right)^{s_{b}^{1} s_{r}^{2}}, R_{r}\right) \cdot e\left(H\left(u_{r}\right), g^{s_{r}^{1} s_{b}^{2}}\right)^{r_{o}} .
\end{aligned}
$$

On the other hand, $u_{r}$ encodes the information he gets into

$$
\begin{aligned}
& e\left(H\left(u_{r}\right)^{s_{b}^{1} s_{a}^{2}}, R_{o}\right) \cdot e\left(H\left(u_{o}\right), g^{s_{a}^{1} s_{b}^{2}}\right)^{r_{r}} ; \\
& e\left(H\left(u_{r}\right)^{s_{b}^{1} s_{c}^{2}}, R_{o}\right) \cdot e\left(H\left(u_{o}\right), g^{s_{c}^{1} s_{b}^{2}}\right)^{r_{r}} ; \\
& e\left(H\left(u_{r}\right)^{s_{b}^{1} s_{o}^{2}}, R_{o}\right) \cdot e\left(H\left(u_{o}\right), g^{s_{o}^{1} s_{b}^{2}}\right)^{r_{r}} .
\end{aligned}
$$

It is clear that encoding (1) is equal to encoding (5). Paths $\left[u_{o}, u_{a}, u_{b}\right]$ and $\left[u_{r}, u_{b}, u_{a}\right]$ compose a 3-depth path between $u_{o}$ and $u_{r}$ (see Figure 2). After the PSI operation, $u_{o}$ and $u_{r}$ are aware that there exists one 3-depth path between them.

4) Discussion: We extend the main idea of $k$-common friends protocol to implement $k$-depth protocol, the two stages of our $k$-depth protocol do not have to be executed sequentially. Path certification stage can be a routine performed by users in the OSN once in a while, e.g., once per month. When $u_{r}$ wants to access $u_{o}$ 's resource, both of them directly execute the path discovery stage, i.e., only $u_{o}$ and $u_{r}$ need to be online in our $k$-depth protocol. As the path certification process is a usual routine, $u_{o}$ and $u_{r}$ cannot agree on some 
nonce, $(k-1)$-depth paths' certificates should be based on common knowledge of users. In our protocol, we use the hash value of user's identity, i.e., $H\left(u_{o}\right)$ and $H\left(u_{r}\right)$. Separation of the two stages also results in efficient communication, i.e., the first stage can be executed when the traffic in OSNs is low, and it also provides better privacy which we explain next.

\section{B. Security Analysis}

There are three parties involving in the protocol including $u_{o}, u_{r}$ and users in the middle. For $u_{o}$ and $u_{r}$, the security goal of our protocol is that we only want them to know whether there is a $k$-depth path between them. Extra knowledge such as who are on the path shouldn't be learned by them. For a user on the path, the security goal of our protocol is that he should only know that he is involving in a path certification stage, he shouldn't know anything more than his friend who sends him the message and the friends he will contact next.

Path certification. In this stage, what each user gets (from messages and reverse messages) are identities, count value, depth, path certificates and stamps. We analyze what information they may leak one by one. For each information, we consider the case under a single as well as multiple $(k-1)$-depth paths' certification processes. To give a clear explanation, we use $k$ users' positions on a $(k-1)$-depth path to represent them. The user on the $i$ th position is denoted by $u_{i}(0 \leq i \leq k-1)$ and $u_{o}$ is at position 0.

Identity. As identities of messages are chosen randomly, they won't leak any information in both single and multiple paths' certification processes.

Count value and depth. In a (k-1)-depth path's certification process, a user gets his position on the path from cnt and the depth of the path from dep. Moreover, when he receives a reverse message from $u_{i+1}$, he knows that $u_{i+1}$ is involved in a $(k-i)$-depth path. We argue that these information are not privacy sensitive, as a user can always guess one of his friend has another friend or a friend of friend.

However, cnt and dep may result in information leakage under several paths' certification processes originated from the same user. A user first sends a message to one of his friends with $c n t$ and $d e p$, later he will know how many (dep-cnt)depth paths this friend originates by counting the number of reverse messages that he gets from this friend. Especially, when $c n t=d e p-1$, he knows how many friends this friend has. This partial structure information can be sensitive in certain cases. To prevent this, each user should send some dummy reverse messages back which produces a noisy version of his social structure.

Path certificates and stamps. Sensitive information in certificates and stamps includes $u_{o}$ 's identity, intermediate users' depth keys and their connections. In a single $(k-1)$-depth path certification, what $u_{i}$ gets are a partial path certificate produced by the first $i$ th users on the path, i.e., $H\left(u_{o}\right)^{\prod_{j=0}^{i-1} s_{j}^{j}}$, the $(k-1)$ depth path's certificate, i.e., $H\left(u_{o}\right)^{\prod_{j=0}^{k-1} s_{j}^{j}}$, and a (partial) path stamp, i.e., $g^{\prod_{j=i+1}^{k-1} s_{j}^{k-j}}$, generated by his successors (from $u_{i+1}$ to $u_{k-1}$ ) on the path.
With $H\left(u_{o}\right)^{\prod_{j=0}^{k-1} s_{j}^{j}}$, if the user is able to get $g^{\prod_{j=0}^{k-1} s_{j}^{j}}$ from another certification process, then by verifying

$$
e\left(H\left(u_{o}\right)^{\prod_{j=0}^{i-1} s_{j}^{j}}, g\right)=e\left(H\left(u_{o}\right), g^{\prod_{j=0}^{i-1} s_{j}^{j}}\right),
$$

he knows who is the owner (through $H\left(u_{o}\right)$ ). Similarly, $H\left(u_{o}\right) \prod_{j=0}^{i-1} s_{j}^{j}$ may also leak $u_{o}$ 's identity with the relative partial path stamp. In Figure 2, $u_{b}$ gets $H(o)^{s_{a}^{1}}$ from path $\left[u_{o}, u_{a}, u_{b}\right]$ 's certification and $g^{s_{a}^{1}}$ from $\left[u_{o}, u_{b}, u_{a}\right]$ 's certification. If $u_{b}$ computes parings of these information with the above equation, he will know that the message sent from $u_{a}$ is originated by $u_{o}$. To prevent this information leakage, $u_{o}$ can send $H\left(u_{o}\right)^{x}$ instead of $H\left(u_{o}\right)$ to $u_{1}$ in the beginning where $x \stackrel{r}{\leftarrow} \mathbb{Z}_{p}^{*}$ is only known to himself and $u_{o}$ generates different $x$ for his different friends. In this way, even an intermediate user gets the corresponding (partial) path stamp, as he knows nothing about $x$, the above equation won't work. Note that before the path discovery stage, $u_{o}$ needs to recover the path certificate with $x^{-1}$.

From $g^{\prod_{j=i+1}^{k-1} s_{j}^{k-j}}$ in the reverse message sent by $u_{i+1}$, due to the hardness of discrete logarithm problem in $G_{1}, u_{i}$ cannot discover $\prod_{j=i+1}^{k-1} s_{j}^{k-j}$. Note that users who happen to be the last one on $(k-1)$-depth paths will expose their "public" 1-depth stamps when they start to forward the reverse messages and these public 1-depth stamps can be treated as their identities. For example, $u_{k-1}$ gets $g^{s_{k-1}^{1}}$ which he can use to identify $u_{k-1}$. However, as a path stamp is computed by users with stamps of different depths, "public" 1-depth stamps will not leak their issuers' identities. For example, suppose that $u_{k-3}$ already knows $g^{s_{k-1}^{1}}$ is from $u_{k-1}$ through another path certification process. When he gets $g^{s_{k-1}^{1} s_{k-2}^{2}}$ from $u_{k-2}$, as he doesn't have $g^{s_{k-2}^{2}}$, he cannot know that $u_{k-1}$ is the last one on the $(k-1)$ depth path (through pairing), i.e., $u_{k-1}$ and $u_{k-2}$ are friends. Moreover, suppose that $u_{k-2}$ even knows that $g^{s_{k-1}^{1}} s_{k-2}^{2}$ is built by $u_{k-2}$ and $u_{k-1}$, he cannot get $u_{k-2}$ 's 2-depth stamp $s_{k-2}^{2}$ from $g^{s_{k-1}^{1} s_{k-2}^{2}}$ and $g^{s_{k-1}^{1}}$ due to the RCDH assumption in $G_{1}$.

Now, suppose that $u_{i}$ and $u_{i+x}(i+x \leq k-1)$ are friends, i.e., a circle appears in the path. When $u_{i}$ joins the process and sends a message to $u_{i+1}, u_{i+1}$ then contacts $u_{i+2}$, so on and so forth. Later, $u_{i+x}$ sends a message to $u_{i}$. Since $u_{i}$ has no information about his successors' depth keys, he doesn't know that the message he receives from $u_{i+x}$ is based on the message he sends to $u_{i+1}$ before. Therefore, $u_{i}$ doesn't know that $u_{i+1}$ and $u_{i+x}$ are linked through a $(x-1)$-depth path.

However, with several path's certification processes, sensitive information can be disclosed through certificates and stamps. Suppose $u_{o}$ 's two friends are linked by a $(k-2)$ depth path, i.e., $u_{o}$ is in a $k$-depth circle. Later, when he gets path certificates and stamps on two $(k-1)$-depth paths which can compose the circle from these two friends, as the circle is also a $k$-depth path, by performing bilinear map, he can get whether these two friends are connected by a $(k-2)$-depth path. In the example above, $u_{o}$ is linked with $\left[u_{o}, u_{a}, u_{b}\right]$ and $\left[u_{o}, u_{b}, u_{a}\right]$, by paring path certificates and stamps on these two paths, he has $e\left(H\left(u_{o}\right)^{s_{a}^{1} s_{b}^{2}}, g^{s_{b}^{1} s_{a}^{2}}\right)=e\left(H\left(u_{o}\right)^{s_{b}^{1} s_{a}^{2}}, g^{s_{a}^{1} s_{b}^{2}}\right)$ which indicates that $u_{a}$ and $u_{b}$ are friends. We propose a 
simple solution for this leakage. Now, the protocol regulates that when a user receives a message with $d e p=2$ and $c n t=1$, he only sends new messages to his friends who haven't sent him a message with $d e p=2$ and $c n t=2$ yet. In Figure 2, after $u_{a}$ and $u_{b}$ receive messages from $u_{o}$, suppose that $u_{a}$ first sends $m_{3}=\left(i_{3}, H(o)^{s_{a}^{1}}, 2,2\right)$ to $u_{b}$. Later, when $u_{b}$ wants to send new messages to his friends, as he finds out that $u_{a}$ already sent him $m_{3}$ with $d e p=2$ and $c n t=2$, he only sends new messages to $u_{c}$ and $u_{r}$. In the end, $u_{o}$ won't know that $u_{a}$ and $u_{b}$ are connected. However, since there is no information in a message about the originator of the path, our solution reduces chances for finding paths. For example, the message sent from $u_{a}$ to $u_{b}$ may come from another user than $u_{o}$. Also, our solution only supports 2-depth path certification for 3-depth policy. Although the information that a user knows his two friends are linked with a 5-depth path is not that valuable, protecting two users' private links under 3 depths is still necessary. We leave the general protection scheme as a future work.

Path discovery. In this stage, only $u_{o}$ and $u_{r}$ participate the protocol. First, as the two stages are independent, no intermediate users knows who is the owner or requester. Moreover, intermediate users do not know if there will be a run of the path discovery stage. Second, as the cardinality PSI protocol is secure against honest but curious adversaries, $u_{o}$ and $u_{r}$ only get whether a qualified path exists or not, nothing more. Especially, $u_{o}$ also doesn't know which friend of his is on the $k$-depth path. The same holds for $u_{r}$.

\section{Multi-relationship k-depth Protocol}

Our $k$-depth protocol can be extended to support multirelationships. We first introduce the multi-relationship social network model. Let the set $\mathcal{R}$ contain all the relationship types. Only symmetric relationships, such as friend and colleague, are considered. The social network is defined as a graph $\mathcal{G}=(\mathcal{U}, \mathcal{E})$, where $\mathcal{E}$ now is denoted as a subset of $\mathcal{U} \times \mathcal{R} \times \mathcal{U}$, i.e., each edge is labeled with a relationship type. A $k$ depth access control policy regulates that $u_{o}$ is linked with $u_{r}$ through a $k$-depth path where each edge has a certain relationship type. All $k$ relationship types (from $u_{o}$ to $u_{r}$ ) can be represented as a $k$-tuple $\left(r p_{1}, r p_{2}, \ldots, r p_{k}\right)$ where $r p_{i} \in \mathcal{R}$ $(1 \leq i \leq k)$. Note that these $k$ relationships do not have to be distinct from each other.

Our multi-relationship $k$-depth protocol also contains two stages. The path discovery stage remain the same while there are two differences related to the path certification stages. First, a relationship chain is added in each message and its function is to inform intermediate users which social links to contact next. A relationship chain generated by $u_{o}$ is defined as $\left\langle r p_{1}, \ldots, r p_{k-1}\right\rangle$ which contains the first $(k-1)$ th relationship types specified in the policy with the same sequence. Moreover, the first relationship in a chain is defined as the tail of the chain. On the other direction, the relationship chain generated by $u_{r}$ is in a reverse order, i.e., $\left\langle r p_{k}, \ldots, r p_{2}\right\rangle$. When a user receives a message, he will delete the tail from the chain and send new messages to his social links who are
TABLE I

COMPARISON OF $k$-DEPTH PROTOCOLS

\begin{tabular}{|c||c|c|c|}
\hline & {$[17]$} & {$[16]$} & Our work \\
\hline \hline Intermediate user offline & $\checkmark$ & & $\checkmark$ \\
\hline Multi-relationships & & & $\checkmark$ \\
\hline Computation cost & Hash & Hash & Paring \\
\hline Communication steps & $k$ & $k$ & $k-1$ \\
\hline Honest but curious model & Partially & Partially & $\checkmark$ \\
\hline
\end{tabular}

in the new tail of the chain with him. The second difference is that we have to integrate the relationship type into paths' certificates and stamps. Instead of one set, each user should have different sets of depth stamps for different relationship types. When a user receives a message, he uses his cnt-depth stamp from the stamp set related to the tail of the chain to compute the new certificate. The same procedure applies for computing the path stamp. Note that the relationship type a user integrates into a path's certificate (stamp) is always the one that he is in with the user who sent him the message (reverse message). This guarantees that encodings related to two $(k-1)$-depth paths that can compose a $k$-depth path in discovery stage are identical.

\section{Comparison with Existing Schemes}

We compare our $k$-depth protocol with the schemes proposed in [17], [16] (see Table I). The solution in [17] and our protocol contain two independent stages, only $u_{o}$ and $u_{r}$ need to be online when finding the path. On the other hand, the protocol proposed in [16] requires all the intermediate users to be online. Different from ours, the two protocols [17], [16] do not support multi-relationships.

Messages passing among users in their schemes are based on hash functions, this is more efficient than the bilinear map. On the other hand, our protocol consumes one step of communications less when finding the paths (each user certifies $(k-1)$ depth paths, instead of $k$-depth) than theirs which save a large number of operations. More precisely, suppose that each user has in average $n$ friends, to find a $(k-1)$-depth path, totally $2(k-1) n^{k-1}$ times user-to-user communications are consumed (see Sect. VII), while the number is $k n^{k}$ in both works. Moreover, as each communication step needs computations, a large number of computations (mainly exponentiations) are saved in our protocol as well.

Since their tokens are built through a publicly known hash function, sensitive information can be leaked. For example, as mentioned in [17], a user can know whether a token he receives is based on another token sent by him previously. This indicates his corresponding two friends are linked. The same threat happens to the scheme in [16]. However, this information leakage can be prevented in our protocol as we explained in the security analysis.

\section{PERformance Analysis}

In this section, we first give a formal efficiency analysis of our protocols, then present empirical results on the protocols through a Facebook dataset [31]. 
TABLE II

THEORETICAL PERFORMANCE ANALYSIS.

\begin{tabular}{|c||c|c|}
\hline Computation & $k$-common fri. & $k$-depth \\
\hline \hline Paring & $4 n$ & $4 n^{k-1}$ \\
\hline Hash & $G_{1}: 2 n$ & $G_{1}: 2 n^{k-1}$ \\
\hline Multiplication & $G_{2}: 2 n$ & $G_{2}: 2 n^{k-1}$ \\
\hline Exponentiation & $G_{2}: 2 n$ & $G_{1}: 2(k-1) n^{k-1} G_{2}: 2 n^{k-1}$ \\
\hline PSI & $O(n \log \log n)$ & $O\left(n^{k-1} \log \log n^{k-1}\right)$ \\
\hline \hline Communication & $k$-common fri. & $k$-depth \\
\hline \hline User-to-user & 2 & $2(k-1) n^{k-1}$ \\
\hline PSI & $O(n)$ & $O\left(n^{k-1}\right)$ \\
\hline
\end{tabular}

\section{A. Theoretical Efficiency Analysis}

For each of our protocols, we analyze its computation and communication complexities (see Table II). We assume that each user's average number of friends is $n$. As friendship establishments are normal routines, we do not consider them as part of our protocols. Moreover, for $k$-common friends and $k$-depth protocols, we exploit the PSI scheme proposed in [32] to give a general complexity for our protocols.

1) Computation Cost: $k$-common friends. Computations are performed in both encoding stage and PSI protocol. To encode a friendship certificate, $u_{o}\left(u_{r}\right)$ needs to perform two pairing computations (each encoding contains two components), one hash function operation in $G_{1}$, one exponentiation and one multiplication in $G_{2}$. Since there are totally $2 n$ friends for $u_{o}$ and $u_{r}, 4 n$ times of parings, $2 n$ hashes in $G_{1}, 2 n$ exponentiations and $2 n$ multiplications in $G_{2}$ are needed. Inputs for PSI operations are $2 n$ encodings, computation related to set intersection can be finished in $O(n \log \log n)$ time [32].

$k$-depth. Since the two stages of our protocol are independent, for the path certification stage, we only consider computation and communication consumption of a single user. In this stage, computations are mainly exponentiations in $G_{1}$ for path certificates and stamps. For a $(k-1)$-depth path staring from $u_{o}$, as $k-1$ users compute the path certificate and stamp by exponentiation, totally $2(k-1)$ exponentiations are needed, i.e., $k-1$ for path certificate and $k-1$ for path stamp. For the whole stage, $u_{o}$ can get maximal $n^{k-1}$ pairs of path certificate and stamp. Therefore, the computation cost for a single user is $2(k-1) n^{k-1}$ exponentiations.

For the path discovery stage, there are maximal $2 n^{k-1}$ path certificates need to be encoded for both $u_{o}$ and $u_{r}$, still each encoding needs two parings, one hash in $G_{1}$, one exponentiation and one multiplication in $G_{2}$. Therefore, totally $4 n^{k-1}$ paring operations, $2 n^{k-1}$ hashes in $G_{1}, 2 n^{k-1}$ exponentiations and $2 n^{k-1}$ multiplications in $G_{2}$ are needed. Again, with $2 n^{k-1}$ path certificates as inputs, by adopting the PSI protocol in [32], computation complexity for finding common encodings is $O\left(n^{k-1} \log \log n^{k-1}\right)$.

2) Communication Cost: $k$-common friends. Communications are needed in two operations. The first one is exchanging two random values in the beginning, where two user-touser communications are needed. The second communication consuming operation is related to the PSI protocol. As the PSI
TABLE III

DATASET SUMMARY.

\begin{tabular}{|c|r|}
\hline Number of nodes & 63,731 \\
\hline Number of edges & $1,634,180$ \\
\hline Average degree & 25.6 \\
\hline Average clustering coefficient & 0.253 \\
\hline Number of connected components & 144 \\
\hline Number of triangles & $3,501,542$ \\
\hline
\end{tabular}

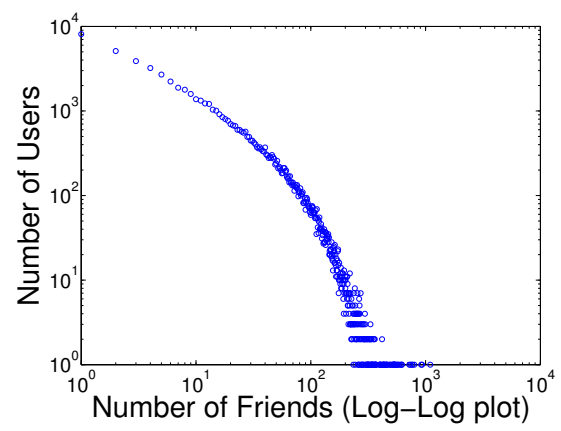

Fig. 3. Node distribution of the dataset.

protocol has constant rounds and its inputs are $2 n$ encodings, it needs $O(n)$ user-to-user communications.

$k$-depth. As mentioned before, a user can get maximal $n^{k-1}$ pairs of path certificate and stamp in path certification stage. Each pair requires $2(k-1)$ user-to-user communications. Totally $2(k-1) n^{k-1}$ communications are needed. Path discovery stage is similar to $k$-common friends protocol, its communication complexity is $O\left(n^{k-1}\right)$.

\section{B. Empirical Efficiency Analysis}

Dataset. We use the Facebook dataset collected by the authors of [31] to perform our experiments, the dataset is summarized in Table III. In Figure 3, we plot the number of users as a function of users' number of friends, as we can see, users' friends number, i.e., degree in social graph, follows a powerlaw distribution. Most of users have a small number of friends (around half of users have less than 10 friends) while only a few users have a large number of connections. This indicates that most of users won't need to perform a huge amount of computations when running our protocols.

Experiment setup. Our experiments were conducted on a 64-bit Linux system with an Intel Core i7 $1.80 \mathrm{GHz} \times 4$ and 8GB RAM. We implement our protocols using the MIRACL Cryptographic SDK [33]. We choose Barreto-Naehrig Curve (security level AES-128) as the pairing curve. Since there are many existing PSI protocols and implementations, we can adopt any of them. For example, the scheme in [25] can perform set intersection operations on two million-element sets within 41 seconds. Therefore, we only focus on the performance of our protocol before the execution of PSI, where pairing operations dominate the computation cost. In our experiments, performing a pairing takes around $6 \mathrm{~ms}$.

We randomly sampled 2,000 users from the dataset for $k$ common friends, 3-depth protocol and 4-depth, respectively. As the average path length between any two users (from more 
TABLE IV

SAMPLE USERS' FEATURE SUMMARY.

\begin{tabular}{|c||r|r|r|r|}
\hline & $k$-common & \multicolumn{1}{c|}{ 3-depth } & \multicolumn{1}{c|}{ 4-depth } & 4-depth (100) \\
\hline \hline Total & 49,665 & $4,398,980$ & $511,424,020$ & $27,163,893$ \\
\hline Average & 24.8 & $2,199.5$ & $255,710.0$ & $271,640.9$ \\
\hline S.D. & 40.7 & $5,067.2$ & $613,000.1$ & $733,250.8$ \\
\hline Max. & 570 & 92,748 & $7,213,810$ & $4,514,853$ \\
\hline
\end{tabular}

than 1 billion users of Facebook) is 4.7 [34], 5-depth protocol is neither necessary nor likely to be performed. Therefore, we only evaluated $k$-depth protocol with $k=3,4$. Moreover, in reality the 3-depth protocol is exploited more often than the 4-depth protocol. The number of friends as well as the number of 2-depth and 3-depth paths for the sampled users are summarized in Table IV. Note that we only chose 100 users to perform 4-depth protocol for the purpose of illustration. These 100 users features are summarized in the last column of Table IV: its mean and standard deviation value are not that different from the sample of 2,000 users for 4-depth protocol. We notice that all the sample data have large std. value, this is due to the power law distribution of users' friends number, see Figure 3.

Evaluation results. The experiment results are presented in Table V. For $k$-common friends, the average time for encoding all friendship certificates is 0.135 second while the worst case (the user who has 570 friends, see Table IV) takes only 3 seconds. Due to the power law distribution of friends number, almost half of users $(48.1 \%)$ can finish their protocol in less than 0.05 second, more than $94 \%$ users can finish in 0.5 second. For 3-depth protocols, the average running time is around 16 seconds. More than half of the 3-depth protocols $(1,121 / 2,000)$ can finish within 5 seconds. The average running time of the 4-depth protocols is about 33.6 minutes. In fact, 34 users (out of 100) can finish their protocol in 60 seconds; nearly half of them (47/100) can finish in less than 3 minutes; and about $70 \%$ of the users can finish in less than 10 minutes. As described in Section VI, path certification can be treated as a normal routine, thus it doesn't have to be counted as part of $k$-depth protocol. Moreover, a user can choose not to join a $k$-depth protocol, or not to send messages to all his friends, meaning that the computations needed in practice can be further reduced. Another way to improve the performance of the $k$-depth protocols is to use more efficient paring implementation, such as the inline assembly code of MIRACL.

Discussion on $k$-depth protocols. As discussed in Section VI, although we use expensive pairing operations, our $k$-depth protocol's performance is still comparable with the protocol proposed in [17] which mainly uses hash functions. First, our $k$-depth protocol uses one less step for communications, thus a big amount of computation overhead can be saved. As presented in Table IV, the total number of 3-depth paths (for the 4-depth protocol) for the sample users is 100 times larger than the number for 2-depth paths (for the 3-depth protocol). Therefore, the scheme in [17] needs to perform at least 100 times more operations, i.e., hash functions as well as communications than ours. Second, in the path certification
TABLE V

TIME CONSUMPTION SUMMARY (SEC).

\begin{tabular}{|c|c|c|c|c|c|}
\hline \multicolumn{7}{|c|}{$k$-common friends } \\
\hline \hline Average & 0.135 & S.D. & 0.222 & Worst case & 3.078 \\
\hline \hline Time & $\leq 0.01$ & $(0.01,0.05]$ & $(0.05,0.1]$ & $(0.1,0.5]$ & $>0.5$ \\
\hline$\%$ users & 13.65 & 34.45 & 14.90 & 31.65 & 5.35 \\
\hline \hline \multicolumn{7}{|c|}{ 3-depth } \\
\hline \hline Average & 16.263 & S.D. & 37.447 & Worst case & 684.401 \\
\hline \hline Time & $\leq 1$ & $(1,5]$ & $(5,10]$ & $(10,20]$ & $>20$ \\
\hline$\%$ users & 30.95 & 25.10 & 11.95 & 11.50 & 20.50 \\
\hline \hline \multicolumn{7}{|c|}{ 4-depth } \\
\hline Average & $2,015.185$ & S.D. & $5,372.101$ & Worst case & $32,833.750$ \\
\hline \hline Time & $\leq 60$ & $(60,180]$ & $(180,600]$ & $(600,1200]$ & $>1200$ \\
\hline$\%$ users & 34.00 & 13.00 & 23.00 & 7.00 & 23.00 \\
\hline
\end{tabular}

stage, intermediate users can choose not to join in our protocol. Hence, the user will get a subset of all the 3-depth paths in the end.

In details, users in the first protocol of [17] need to build an "imaginary" hash tree, and the number of descendants of each node is the number of maximal degree of a user in the social network. This will be a huge tree with a lot of redundant nodes. In the dataset that we use, the maximal node degree is 1,098 , meaning that the tree a user needs to build for a 3depth protocol will have $\mid u$.fri $\mid \times 1098 \times 1098$ nodes, while in our experiments each user only needs to perform around 2,200 times pairing products on average. For example, for a user with only 25 friends, to perform $25 \times 1098 \times 1098$ times SHA-256 function in MIRACL, it needs around 29 seconds while in our scheme the computation only needs 16 seconds. In the extended scheme of [17], the user can build a more accurate hash tree. However, this extended scheme is only designed for discovering 3-depth paths.

\section{CONCLUSION}

In this paper, we addressed the challenge on how to enforce relationship-based access control policies on decentralized social networks. To this end, we have provided privacypreserving protocols for two types of access control policies, i.e., $k$-common friends and $k$-depth. While the protocol for $k$-common friends is new, our $k$-depth protocol has better communication complexity and security than the existing solutions. Through experiments on a Facebook dataset, we illustrate that our protocols are efficient in practice.

There are a few research directions for the future. Firstly, it is interesting for us to re-evaluate the performance of our protocols with some more recent social network dataset than the current dataset [31] used in this paper. Secondly, we want to make our $k$-depth protocol secure under the malicious adversary model [26] and design protocols for other policies such as 'clique' and 'celebrity' [4]. It is also important for us to investigate whether it is possible to extend our protocols to cope with friendship revocation by incorporating some ideas, for example, from [35], [36]. 


\section{ACKNOWLEDGMENT}

We would like to thank Qiang Tang for his insightful comments.

\section{REFERENCES}

[1] P. W. Holland and S. Leinhardt, "Transitivity in structural models of small groups." Comparative Group Studies, 1971.

[2] C. E. Gates, "Access control requirements for Web 2.0 security and privacy," in Proc. IEEE Workshop on Web2.0 Security and Privacy (W2SP), 2007.

[3] B. Carminati, E. Ferrari, R. Heatherly, M. Kantarcioglu, and B. Thuraisingham, "A semantic web based framework for social network access control," in Proc. 14th ACM Symposium on Access Control Models and Technologies (SACMAT). ACM, 2009, pp. 177-186.

[4] P. W. L. Fong, M. M. Anwar, and Z. Zhao, "A privacy preservation model for Facebook-style social network systems," in Proc. 14th European Symposium on Research in Computer Security (ESORICS), ser. LNCS, vol. 5789. Springer, 2009, pp. 303-320.

[5] S.-W. Seong, J. Seo, M. Nasielski, D. Sengupta, S. Hangal, S. K. Teh, R. Chu, B. Dodson, and M. S. Lam, "PrPl: A decentralized social networking infrastructure," in Proc. 1st ACM Workshop on Mobile Cloud Computing \& Services: Social Networks and Beyond. ACM, 2010, pp. $1-8$.

[6] L. A. Cutillo, R. Molva, and M. Önen, "Safebook: A distributed privacy preserving online social network," in Proc. 12th IEEE International Symposium on a World of Wireless, Mobile and Multimedia Networks. IEEE CS, 2011, pp. 1-3.

[7] C.-m. A. Yeung, I. Liccardi, K. Lu, O. Seneviratne, and T. BernersLee, "Decentralization: The future of online social networking," in W3C Workshop on the Future of Social Networking Position Papers, vol. 2, 2009 , pp. 2-7.

[8] B. Carminati, E. Ferrari, and A. Perego, "Enforcing access control in web-based social networks," ACM Transactions on Information \& System Security, vol. 13, no. 1, p. Article No. 6, 2009.

[9] P. W. L. Fong and I. Siahaan, "Relationship-based access control policies and their policy languages," in Proc. 16th ACM Symposium on Access Control Models and Technologies (SACMAT). ACM, 2011, pp. 51-60.

[10] P. W. L. Fong, "Relationship-based access control: protection model and policy language," in Proc. 1st ACM Conference on Data and Application Security and Privacy (CODASPY). ACM, 2011, pp. 191-202.

[11] G. Bruns, P. W. L. Fong, I. Siahaan, and M. Huth, "Relationship-based access control: its expression and enforcement through hybrid logic,' in Proc. 2nd ACM Conference on Data and Application Security and Privacy (CODASPY). ACM, 2012, pp. 117-124.

[12] J. Pang and Y. Zhang, "A new access control scheme for Facebook-style social networks," in Proc. 9th Conference on Availability, Reliability and Security (ARES). IEEE CS, 2014, pp. 1-10.

[13] Y. Cheng, J. Park, and R. S. Sandhu, "Relationship-based access control for online social networks: beyond user-to-user relationships," in Proc. 4th IEEE Conference on Information Privacy, Security, Risk and Trust (PASSAT). IEEE CS, 2012, pp. 646-655.

[14] B. Carminati and E. Ferrari, "Privacy-aware collaborative access control in web-based social networks," in Proc. 22nd IFIP WG 11.3 Working Conference on Data and Applications Security (DBSEC), ser. LNCS, vol. 5094. Springer, 2008, pp. 81-96.

[15] _ - "Enforcing relationships privacy through collaborative access control in web-based social networks," in Proc. 5th Conference on Collaborative Computing (CollaborateCom). IEEE CS, 2009, pp. 1-8.

[16] M. Xue, B. Carminati, and E. Ferrari, "P3D - privacy-preserving path discovery in decentralized online social networks," in Proc. 35th IEEE Computer Software and Applications Conference (COMPSAC). IEEE CS, 2011, pp. 48-57.

[17] G. Mezzour, A. Perrig, V. Gligor, and P. Papadimitratos, "Privacypreserving relationship path discovery in social networks," in Proc. 8th Conference on Cryptology and Network Security (CANS), ser. LNCS, vol. 5888. Springer, 2009, pp. 189-208.

[18] M. Backes, M. Maffei, and K. Pecina, "A security API for distributed social networks," in Proc. 18th Annual Network \& Distributed System Security Symposium (NDSS). Internet Society, 2011, pp. 35-51.

[19] K. B. Frikken and P. Srinivas, "Key allocation schemes for private social networks," in Proc. 8th ACM Workshop on Privacy in the Electronic Society (WPES). ACM, 2009, pp. 11-20.
[20] X. Chen, F. Zhang, and K. Kim, "A new ID-based group signature scheme from bilinear pairings," in IACR ePrint Archive: Report 2003/116, 2003

[21] D. Boneh, B. Lynn, and H. Shacham, "Short signatures from the Weil pairing," in Proc. 7th Conference on the Theory and Application of Cryptology and Information Security (ASIACRYPT), ser. LNCS, vol. 2248. Springer, 2001, pp. 514-532.

[22] M. Bellare and P. Rogaway, "Random oracles are practical: a paradigm for designing efficient protocols," in Proc. 1st ACM Conference on Computer and Communications Security (CCS). ACM, 1993, pp. 6273.

[23] M. J. Freedman, K. Nissim, and B. Pinkas, "Efficient private matching and set intersection," in Proc. 23rd Conference on the Theory and Applications of Cryptographic Techniques (EUROCRYPT), ser. LNCS, vol. 3027. Springer, 2004, pp. 1-19.

[24] E. Stefanov, E. Shi, and D. Song, "Policy-enhanced private set intersection: sharing information while enforcing privacy policies," in Proc. 15th Conference on Practice and Theory in Public Key Cryptography $(P K C)$, ser. LNCS, vol. 7293. Springer, 2012, pp. 413-430.

[25] C. Dong, L. Chen, and Z. Wen, "When private set intersection meets big data: an efficient and scalable protocol," in Proc. 20th ACM Conference on Computer and Communications Security (CCS). ACM, 2013, pp. 789-800.

[26] O. Goldreich, The Foundations of Cryptography - Volume 2. Cambridge University Press, 2004

[27] A. C. Squicciarini, M. Shehab, and F. Paci, "Collective privacy management in social networks," in Proc. 18th Conference on World Wide Web $(W W W)$. ACM, 2009, pp. 521-530.

[28] Y. Sun, C. Zhang, J. Pang, B. Alcalde, and S. Mauw, "A trust-augmented voting scheme for collaborative privacy management," in Proc. 6th Workshop on Security and Trust Management (STM), ser. LNCS, vol. 6710. Springer, 2011, pp. 132-146.

[29] _ - "A trust-augmented voting scheme for collaborative privacy management," Journal of Computer Security, vol. 20, no. 4, pp. 437-459, 2012.

[30] H. Hu, G.-J. Ahn, and J. Jorgensen, "Multiparty access control for online social networks: model and mechanisms," IEEE Transactions on Knowledge and Data Engineering, vol. 10, no. 6, pp. 341-354, 2013.

[31] B. Viswanath, A. Mislove, M. Cha, and K. P. Gummadi, "On the evolution of user interaction in facebook," in Proc. 2nd ACM SIGCOMM Workshop on Social Networks (WOSN). ACM, 2009, pp. 37-42.

[32] C. Hazay and K. Nissim, "Efficient set operations in the presence of malicious adversaries," in Proc. 13th Conference on Practice and Theory in Public Key Cryptography (PKC), ser. LNCS, vol. 6056. Springer, 2010, pp. 312-331

[33] "Miracl crypto library," http://www.certivox.com/miracl/.

[34] J. Ugander, B. Karrer, L. Backstrom, and C. Marlow, "The anatomy of the facebook social graph," CoRR, vol. abs/1111.4503, 2011.

[35] S. Jahid, P. Mittal, and N. Borisov, "EASiER: Encryption-based access control in social networks with efficient revocation," in Proc. 6th ACM Symposium on Information, Computer and Communications Security (ASIACCS). ACM, 2011, pp. 411-415.

[36] S. Preibusch and A. R. Beresford, "Establishing distributed hidden friendship relations," in Proc. 17th Workshop on Security Protocols $(S P W)$, ser. LNCS, vol. 7028. Springer, 2013, pp. 321-334. 